\title{
Validating the Unified Theory of Acceptance and Use of Technology (UTAUT) tool cross-culturally
}

\author{
Lidia Oshlyansky \\ University of Wales Swansea \\ Singleton Park \\ Swansea SA2 8PP \\ +44 1792205678 \\ lidiaosh@gmail.com
}

\author{
Paul Cairns \\ Dept of Computer Science \\ University of York \\ York Y010 5DD \\ +44 1904434336 \\ pcairns@cs.york.ac.uk
}

\author{
Harold Thimbleby \\ University of Wales Swansea \\ Singleton Park \\ Swansea SA2 8PP \\ +44 1792205678 \\ harold@thimbleby.net
}

\begin{abstract}
HCI methods and tools are often used cross-culturally before being tested for appropriateness and validity. As new tools emerge, they must be cross-culturally validated to ensure that they work with all audiences, not just those in the country in which they were developed. This paper presents the validation of a technology acceptance model over nine culturally-diverse countries. The model validated is the Unified Theory of Acceptance and Use of Technology (UTAUT). The paper also explores ongoing analysis of the culture differences that emerge on UTAUT measures, and suggests avenues for future work.
\end{abstract}

\section{Categories and Subject Descriptors}

H.5.2 [User interfaces]: Evaluation/methodology

\section{General Terms}

Measurement, Reliability, Standardization, Verification.

\section{Keywords}

Cross-cultural, technology acceptance, UTAUT, validation.

\section{INTRODUCTION}

Particularly with website design and mobile devices, HumanComputer Interaction practitioners face the challenges of designing across cultures daily, but there is little in the way of proven, cross-culturally validated tools. Ongoing research has shown that differences do exist in the way subjects in different cultures respond to standard usability measurement techniques [3,6]. Standard HCI measurement tools have been shown to have cultural differences that can be missed if they are not carefully evaluated and considered [13].

HCI has often relied on cultural models to help explain differences found in various aspects of usability and interaction. For example, cultural models have been used to explain crosscultural differences in technology acceptance, adoption and uptake $[2,5,10]$. However, models of cultural differences may not be applicable, or indeed valid, for use in the field $[9,11,14]$.

(C) Oshlyansky, Cairns, Thimbleby 2007 Published by the British Computer Society Volume 2 Proceedings of the 21st BCS HCI Group Conference

HCI 2007, 3-7 September 2007, Lancaster University, UK Devina Ramduny-Ellis \& Dorothy Rachovides (Editors)
“Technology acceptance," that is, people's attitude to the uptake and use of different technologies, has emerged as a strong candidate for cross-cultural validation of HCI tools. Previous research has undertaken some work to cross-culturally validate and culturally extend technology acceptance models, such as the Technology Acceptance Model (TAM) [4,7,15]. However, the Unified Theory of Acceptance and Use of Technology, UTAUT [16], is a more recent instrument, which is a synthesis of eight existing models of technology acceptance - including TAM. UTAUT also integrates elements from: Theory of Reasoned Action, Motivational Model, Theory of Planned Behaviour (TPB), a combined TAM and TPB model, Model of PC Utilization, Innovation Diffusion Theory, and Social Cognition Theory. The unification of these models provides UTAUT with eight constructs: Performance expectancy, Effort expectancy, Attitude towards using technology, Social influence, Facilitating conditions, Self-efficacy, Anxiety and Behavioural intention to use the system [16].

UTAUT has already been validated and applied in the field in English-speaking countries $[1,16]$. The present paper provides a cross-cultural validation.

\section{PREPARING UTAUT}

The aim of the present study was to collect data from countries around the world to cross-culturally validate the UTAUT tool. The data was collected from undergraduate and postgraduate students from all countries sampled. To make the questions on UTAUT accessible and applicable to all participants in the study, the general use of websites was queried, which ensured that all participants would have access to the technology in question. External factors, such as price and technology availability, would not interfere or skew confound the findings.

UTAUT is meant to be adjusted to fit the technology being queried [16], and therefore a certain amount of rewording is expected. Due to the nature of the questions being asked, and because this research did not seek to predict usage or acceptance of a particular application, certain measures on UTAUT were excluded. Behavioural intention to use was dropped, as it is intended as a predictor of use. Facilitating conditions was excluded specifically because the chosen technology, websites, would be available and accessible to all participants - making many of the questions on this construct redundant. The question, "Using the system is a bad/good idea" was dropped from Attitude towards using technology construct as it was not possible to make its wording suitable for the general question of website use.

These adjustments may of course influence the validity of the instrument, as measures are removed and questions changed. 
To ensure that these changes have not affected the overall validity of UTAUT, an analysis of the data from English only speaking countries was done. As will be discussed in the Results section of this paper, these changes did not significantly influence the tool.

\subsection{Translation}

Once all questions were reworded, UTAUT was translated into six languages: Arabic (Saudi Arabian), Czech, Dutch, French, Greek, and Malay. Each translation was completed by at least two bilingual speakers, using the back-translation process. This process ensures that meaning and nuance are not lost, and that the translated versions of the questionnaires remain as true to the original as possible [12]. The translation process did not give rise to further modifications of the UTAUT tool.

\section{PARTICIPANTS}

Table 1. Per country participant sample summary

\begin{tabular}{|lcccccc|}
\hline & $\begin{array}{c}\text { Total } \\
\text { sample }\end{array}$ & $\begin{array}{c}\text { Reject } \\
\text { data }\end{array}$ & $\begin{array}{c}\text { Used } \\
\text { sample }\end{array}$ & Male & Female & $\begin{array}{c}\text { Mean } \\
\text { age }\end{array}$ \\
\hline Czech & 157 & 5 & 152 & 98 & 44 & 23.3 \\
Republic & & & & & & \\
Greece & 152 & 36 & 116 & 39 & 61 & 23.75 \\
India & 129 & 36 & 93 & 68 & 21 & 23.44 \\
Malaysia & 187 & 19 & 168 & 49 & 102 & 21.99 \\
New & 199 & 92 & 107 & 58 & 48 & 21.7 \\
Zealand & & & & & & \\
Saudi & 123 & & 91 & 36 & 46 & 24.19 \\
Arabia & & & & & & \\
South & 144 & 34 & 110 & 75 & 27 & 20.94 \\
Africa & & & & & & \\
United & 242 & 125 & 117 & 53 & 61 & 25.68 \\
Kingdom & & & & & & \\
United & 156 & 30 & 126 & 43 & 79 & 24.28 \\
States & 1489 & 286 & 1080 & 519 & 489 & 23.25 \\
\hline Totals & 145 \\
\hline
\end{tabular}

The translated questionnaires were distributed to university students in the Czech Republic, France, Greece, India, Malaysia, Netherlands, New Zealand, Saudi Arabia, South Africa, the United Kingdom, and to the United States. In all countries students were recruited from diverse Faculties including, Humanities, Science, Health Science, Medicine, Engineering, Computer Science, Business and Economics. No sample was represented by less than 5 academic disciplines.

A total of 1,570 questionnaires were returned. Only those countries returning close to 100 or more questionnaires were left in for analysis (table 1). This meant that the France $(N=38)$ and the Netherlands $(N=43)$ were not used in further analysis. Of the remaining sample, participants were kept in the analysis if their questionnaires were completed and no suspect data pattern was present; that is, questionnaires with more than five questions missing, patterns like 1234512345 , or giving all one answer were discarded. Likewise, only native participants, those receiving primary, secondary and university education in the same country as where they were living, were used in the analysis to ensure a truly representative, homogeneous country sample. The sample was matched for age, education and access to technology and was equally balanced by gender. Table 1 summarises the data collected for those countries meeting the selection requirements.

\section{DATA ANALYSIS}

Large sets of data, such as the one collected here, can be difficult to understand without tools that assist in simplifying and summarising them. Factor analysis simplifies a matrix of correlations into more easily comprehensible factors. Factors, in turn, represent a summary of the relationship between sets of variables. Principal Component Analysis (PCA) was used here as it is a good method for exploring broad questions about the relationship between variables in large sets of data. Additionally, PCA makes no distributional assumptions about the data arising from the UTAUT cross-culturally unlike confirmatory factor analysis methods. If the UTAUT constructs are working across cultures - and indeed measuring technology acceptance - they will emerge as one omnibus factor in the analysis [16]. Variables measuring each individual construct should also group together on factors, showing that they measure a particular aspect of technology acceptance [8].

The determination of factors is not defined by the factor analysis method, but instead must be a result of judgment or heuristics. We have used the common guidelines [8] that factors are selected if their eigenvalues are 1 or more; variables having a loading of magnitude 0.3 or more are considered to have a significant influence on the factor especially in large samples such as this. Of course, where these heuristics are nearly met, we highlight this to give a more rounded and flexible picture.

Table 2: UTAUT Principal Component Analysis all countries

\begin{tabular}{|lcccccc|}
\hline & $\mathbf{1}$ & $\mathbf{2}$ & $\mathbf{3}$ & $\mathbf{4}$ & $\mathbf{5}$ & $\mathbf{6}$ \\
Performance & $\mathbf{0 . 3 2}$ & $\mathbf{- 0 . 3 1}$ & $\mathbf{0 . 5 7}$ & 0.07 & 0.15 & 0.02 \\
Performance & $\mathbf{0 . 3 7}$ & $\mathbf{- 0 . 4 2}$ & $\mathbf{0 . 5 4}$ & 0.11 & 0.22 & 0.05 \\
Performance & $\mathbf{0 . 4 3}$ & $\mathbf{- 0 . 3 8}$ & $\mathbf{0 . 5 7}$ & 0.15 & 0.15 & 0.04 \\
Performance & $\mathbf{0 . 4 9}$ & $\mathbf{- 0 . 3 2}$ & $\mathbf{0 . 4 3}$ & 0.16 & -0.01 & 0.04 \\
Effort & $\mathbf{0 . 5 3}$ & $\mathbf{- 0 . 3 8}$ & -0.17 & 0.05 & 0.22 & -0.05 \\
Effort & $\mathbf{0 . 6 5}$ & -0.18 & $\mathbf{- 0 . 3 7}$ & 0.05 & $\mathbf{0 . 3 2}$ & -0.19 \\
Effort & $\mathbf{0 . 6 5}$ & -0.23 & $\mathbf{- 0 . 3 9}$ & 0.07 & $\mathbf{0 . 3 4}$ & -0.21 \\
Effort & $\mathbf{0 . 6 5}$ & -0.18 & $\mathbf{- 0 . 3 6}$ & 0.07 & $\mathbf{0 . 3 2}$ & -0.22 \\
Attitude & $\mathbf{0 . 5 4}$ & -0.17 & 0.02 & $\mathbf{0 . 4 3}$ & $\mathbf{- 0 . 3 2}$ & 0.09 \\
Attitude & $\mathbf{0 . 5 9}$ & -0.07 & -0.22 & $\mathbf{0 . 4 4}$ & $\mathbf{- 0 . 4 4}$ & 0.13 \\
Attitude & $\mathbf{0 . 6 2}$ & -0.10 & -0.25 & $\mathbf{0 . 3 7}$ & $\mathbf{- 0 . 3 9}$ & 0.09 \\
Social & $\mathbf{0 . 6 2}$ & 0.14 & 0.15 & -0.22 & $\mathbf{- 0 . 3 1}$ & $\mathbf{- 0 . 3 7}$ \\
Social & $\mathbf{0 . 6 1}$ & 0.17 & 0.14 & -0.14 & $\mathbf{- 0 . 3 6}$ & $\mathbf{- 0 . 3 3}$ \\
Social & $\mathbf{0 . 6 5}$ & 0.14 & 0.17 & $\mathbf{- 0 . 4 0}$ & -0.18 & -0.15 \\
Social & $\mathbf{0 . 6 3}$ & 0.14 & 0.15 & $\mathbf{- 0 . 4 9}$ & -0.08 & -0.08 \\
Self-efficacy & $\mathbf{0 . 6 3}$ & -0.10 & -0.20 & -0.18 & 0.08 & 0.08 \\
Self-efficacy & $\mathbf{0 . 6 6}$ & 0.22 & -0.08 & -0.17 & 0.01 & $\mathbf{0 . 3 0}$ \\
Self-efficacy & $\mathbf{0 . 5 8}$ & 0.04 & -0.06 & -0.29 & 0.06 & $\mathbf{0 . 4 6}$ \\
Self-efficacy & $\mathbf{0 . 6 0}$ & 0.12 & -0.11 & -0.23 & 0.03 & $\mathbf{0 . 4 8}$ \\
Anxiety & $\mathbf{0 . 4 0}$ & $\mathbf{0 . 6 2}$ & 0.12 & 0.21 & 0.10 & -0.07 \\
Anxiety & 0.19 & $\mathbf{0 . 6 8}$ & 0.14 & 0.20 & 0.26 & 0.02 \\
Anxiety & 0.27 & $\mathbf{0 . 7 7}$ & 0.12 & 0.24 & 0.16 & 0.04 \\
Anxiety & 0.29 & $\mathbf{0 . 7 4}$ & 0.13 & 0.22 & 0.17 & -0.07 \\
\hline
\end{tabular}

\subsection{Analysis of UTAUT in all countries}

The data for the English only language sample was analysed to ensure that the changes made to UTAUT had not affected its overall validity. This sample included New Zealand, the United Kingdom and the United States. South Africa and India were not included in this analysis because while English is used in 
higher education and in business it is not necessarily the only or first language of the participants. The component matrix for this analysis is not reproduced here, as it is very similar to the one presented for all countries in Table 2. Just as in the original devising and evaluation of UTAUT [16], the English-only data produces the first omnibus factor but with the exception of Anxiety.

The data was then analysed as one complete set to ascertain if UTAUT would work on a widely-heterogeneous sample. Table 2 presents the component matrix for the all-countries data set. The questions for each construct appear in the order in which they are presented in the questionnaire. The heading for each column lists the factor number, and the amount of variance account for that factor in brackets. The six factors selected account for over $66 \%$ of the overall variance in the data collected.

The analysis clearly shows the first factor is the general UTAUT factor. All UTAUT constructs load on factor 1, with the exception of Anxiety, which loads strongly on factor 2 (Table 2). Closer examination shows that, in fact, a further two of the Anxiety questions load almost at the 0.3 cut-off point in the all-countries analysis. Thus, we have confidence that UTAUT is working as expected. The only concerning question then is, why the Anxiety measure does not load on factor 1 in the English only analysis.

Table 3: UTAUT constructs emerging on factors 2, 3, 4

\begin{tabular}{|c|c|c|c|}
\hline & Factor 2 & Factor 3 & Factor 4 \\
\hline Czech & Attitude - & Attitude - & Attitude \\
\hline Republic & Effort & $\begin{array}{l}\text { Effort - } \\
\text { Performance } \\
\text { Social }\end{array}$ & Performance \\
\hline Greece & $\begin{array}{l}\text { Anxiety - } \\
\text { Performance }\end{array}$ & $\begin{array}{l}\text { Effort - } \\
\text { Performance }\end{array}$ & Attitude \\
\hline India & Anxiety & --- & Attitude \\
\hline Malaysia & $\begin{array}{l}\text { Attitude - } \\
\text { Anxiety }\end{array}$ & $\begin{array}{l}\text { Attitude } \\
\text { Performance }\end{array}$ & Social - \\
\hline $\begin{array}{l}\text { New } \\
\text { Zealand }\end{array}$ & Anxiety & $\begin{array}{l}\text { Effort } \\
\text { Performance - }\end{array}$ & $\begin{array}{l}\text { Attitude } \\
\text { Performance }\end{array}$ \\
\hline $\begin{array}{l}\text { Saudi } \\
\text { Arabia }\end{array}$ & $\begin{array}{l}\text { Anxiety } \\
\text { Social }\end{array}$ & $\begin{array}{l}\text { Effort } \\
\text { Performance }\end{array}$ & -- \\
\hline $\begin{array}{l}\text { South } \\
\text { Africa }\end{array}$ & $\begin{array}{l}\text { Anxiety - } \\
\text { Performance }\end{array}$ & $\begin{array}{l}\text { Anxiety } \\
\text { Effort - } \\
\text { Performance }\end{array}$ & Attitude - \\
\hline \begin{tabular}{|l} 
United \\
Kingdom
\end{tabular} & $\begin{array}{l}\text { Anxiety - } \\
\text { Effort }\end{array}$ & $\begin{array}{l}\text { Effort - } \\
\text { Performance }\end{array}$ & Attitude \\
\hline $\begin{array}{l}\text { United } \\
\text { States }\end{array}$ & Anxiety & Performance & $\begin{array}{l}\text { Attitude } \\
\text { Social - }\end{array}$ \\
\hline
\end{tabular}

In the remaining factors, questions that measure UTAUT constructs (Performance, Effort Expectancy, Attitude, Social, Self-efficacy, and Anxiety) load, for the most part, in their sets. For example, Performance and Anxiety load on factor 2, Performance also loads with Effort on factor 3. However, in both Effort and Self-Efficacy, there is one question that does not load with the other questions on that scale. The Social and Self Efficacy constructs are both noted as being slightly awkward [16]. The Social construct is strongly influenced by gender, age and experience, while the effect of the Self-efficacy construct is partially captured by the Effort Expectancy construct [16]; this could be the reason for their lower loadings here. In the case of Effort, the missing question is loading factor 2 with the Performance measure. In the English only sample the missing Effort question falls just short of the .30 cut off (.25) on factor 2. (This question rates, "Interacting with the website is clear and understandable.") In the case of the data collected here it seems that this question groups with those of Performance rather than Effort. This behaviour could be an artefact of the technology (the web) being queried and not of the UTAUT tool: people may relate to websites differently than other technologies, especially those that are not as ubiquitous and readily available.

The worst construct is Social. After loading on the omnibus factor 1 , the Social construct is broken up over factors 4, 5 and 6. This may reflect Ventkatesth et al.'s original problems with the Social construct. Overall, then, this analysis of the entire dataset provides some confidence that the UTAUT tool works cross-culturally.

\subsection{UTAUT analysis by country}

An analysis of UTAUT country-by-country provided further evidence that the questionnaire is working as intended in each of the sample countries. Furthermore, translation did not hinder the performance of UTAUT. Factor 1 emerged as the omnibus factor in all countries, except India. In India the sample was small and male-dominant, which may account for the unexpected UTAUT performance in that sample.

As the first factor to emerge is always the UTAUT factor, it is most interesting to consider the remaining factors. Looking at the different UTAUT constructs that appear for each country on the subsequent factors provides a better understanding of specific cultural influence on technology acceptance. Instead of reproducing the component matrices for each country, Table 3 provides a summary of the UTAUT constructs that emerged on factors 2, 3 and 4 for each country. A minus sign after a construct indicates that it loads negatively on the factor rather than positively. The "---" indicates that no clear construct emerged on these factors for the country but rather the factor was made up of different questions from several different constructs.

Table 3 provides clear evidence that the UTAUT constructs continue to load together and work across the nine cultures sampled, even when the countries are examined independently. However, different constructs have different amounts of influence in each country. For example, the Social construct only emerges on factor 2 for the Saudi Arabia sample, which seems to indicate that social influence has greater weight on website acceptance in Saudi Arabia than in the other countries sampled. Anxiety, on the other hand, emerges on factor 2 in all countries except the Czech Republic. This may show that Anxiety is not a strong influence on website acceptance in the Czech Republic. The Effort and Performance constructs almost always emerge on factors 2 and 3 for all the countries (except India, whose sample was problematic). This could point to the overall, cross-cultural importance of these two constructs. The confused factors for India and Saudi Arabia may mean that in these countries UTAUT is not working cleanly or be due to the relatively small sample sizes of India and Saudi Arabia.

Table 4: Kruskal-Wallis test for UTAUT constructs

\begin{tabular}{|lrrc|}
\hline & Chi-Squared & DF & Asymp. Sig. \\
Performance & 76.25 & 8 & r0.000 \\
Effort & 144.21 & 8 & 0.000 \\
Attitude & 189.27 & 8 & 0.000 \\
Social & 145.22 & 8 & 0.000 \\
Self-efficacy & 158.59 & 8 & 0.000 \\
Anxiety & 174.43 & 8 & 0.000 \\
\hline
\end{tabular}




\subsection{Analysis of means of UTAUT constructs}

Because different UTAUT constructs emerged as having more influence (explaining more variance) in some countries sampled than others, it is interesting to see if the differences are significant. It was not possible to assume that the samples were parametric in nature, so the more conservative Kruskal-Wallis test was run instead of ANOVAs. Table 4 gives the results for the Kruskal-Wallis test, and shows that all the mean differences for each of the UTAUT constructs was significant in the nine countries presented here. However, given the large sample sizes used, this result should be interpreted with some caution. Large sample sizes, like the one here, can show significant differences even when these are not entirely interesting or meaningful. Further research and analysis, such as Kruskall-Wallis post tests, is needed to determine the direction of the differences, establish if the differences found here on UTAUT are meaningful to cross-cultural acceptance, and can be translated into guidelines or recommendations for design.

\section{DISCUSSION}

The results presented here clearly show that the UTAUT tool is robust enough to withstand translation and to be used crossculturally, outside its original country and language of origin. This finding is useful, as it enables HCI researchers and practitioners to use the tool in an international context without concern for its cross-cultural validity. Our analysis seems to indicate that the UTAUT tool will uncover cultural differences at least in the constructs it measures. Our analysis also gives an initial indication that the UTAUT tool may be useful in providing insight into cross-cultural technology acceptance differences. This is a particularly useful result, as the current trend for explaining such differences relies heavily on the use of cultural models that have not been validated in the HCI field [11]. Future analysis of the tool would be useful with more and different countries as those covered here are by no means exhaustive.

The Anxiety measure not loading on the omnibus factor could be caused by several aspects of the research. It could be an artefact of the changes made to the UTAUT questionnaire; it could be because participants are asked to reflect on their previous decisions and use, and are therefore emphasising their anxiety more in hindsight; it could be caused by some particular aspect of how people reflect on the use of websites as opposed to other technologies. All these possibilities would benefit from further and more in-depth exploration.

Alternatively, the problem with Anxiety may only be due to one question, since the others all load on the omnibus factor at or around the 0.3 level. This is similar to the exceptional questions in the Effort and Self-Efficacy constructs. It is possible that all these slight anomalies are due to the rewording and realignment of the UTAUT tool to query website use retrospectively. If the tool was used as a predictive measure of acceptance, the concern of the missing questions may not be an issue. It is likely that the missing questions simply do not hold as much sway or as much explanatory power when being used with a technology such as websites. If the tool were used with the intent to query the acceptance of a specific office software application, say, or even a specific web site the concerns noted here may not arise.

\section{ACKNOWLEDGMENTS}

The authors would like to thank Dr. V. Venkatesh for allowing the use of the UTAUT. A great amount of gratitude is owed to those who helped translate and gather data in the countries sampled, unfortunately too numerous to list here; thank you!

\section{REFERENCES}

[1] Anderson, J. and Schwager, P., SME Adoption of Wireless LAN Technology: Applying the UTAUT Model. Proceedings 7th Conference of the Southern Association for Information Systems (SAIS), 39-43, 2004.

[2] Barnett, G. A. \& Sung, E., Culture and the Structure of the International Hyperlink Network. Journal of ComputerMediated Communication. 11(1), 217-238, 2005.

[3] Cleary, Y., The impact of subjective cultural issues on the usability of a localized Web site: The Louvre Museum Web site. In Bearman, D. and Trant, J. (eds.) Museums and the Web 2000. 153-162, 2000.

[4] Day, D., Cultural Bases of interface acceptance: foundations. Proc. of BCS HCI’96. 35-47, 1996.

[5] De Angeli, A., Athavankar, U., Joshi, A., Coventry, L. and Johnson, G. I., Introducing ATMs in India: A contextual inquiry. Interacting with Computers. 16(1), 29-44, 2004.

[6] Evers, V., Cross-Cultural Applicability of User Evaluation Methods: A Case Study amongst Japanese, North American, English and Dutch Users. CHI'O2 extended abstracts on Human Factors in computing systems. 740 741, 2002.

[7] Evers, V. and Day, D., The role of culture in interface acceptance. In Howard, S., Hammond, J. and Lindegaard, G. (eds), Proc. of INTERACT'97, 260-267, 1997.

[8] Kline, P., An Easy Guide to Factor Analysis. Routledge, New York, 2002.

[9] Kruger, T. and Roodt, G., Hofstede’s VSM-94 Revisited: Is it reliable and valid? South African Journal of Industrial Psychology. 29(1), 75-82, 2003.

[10] Maitland, C. F. and Bauer, J. M., National level culture and global diffusion: The case of the Internet. In Ess, C. \& Sudweeks, F. (eds.), Culture, Technology, Communication Towards an Intercultural Global Village. State University of New York Press, Albany, NY, 87-128, 2001.

[11] Oshlyansky, L., Cairns P. and Thimbleby, H., A cautionary tale: Hofstede's VSM revisited. Proc. of BCS HCI 2006. vol 2, 11-15 2006.

[12] Neuman, W. L., Social Research methods: qualitative and quantitave approaches 4th edition. Allyn and Bacon, Boston, 2000.

[13] Shimaneni, J. S. and Dunckley, L., Users' Satisfaction An African Perspective. Proc. of BCS HCI, vol 2, 167-172. 2005.

[14] Spector, P. E., Cooper, C. L. and Sparks, K., An International Study of the Psychometric Properties of the Hofstede Value Survey Module 1994: A Comparison of Individual and Country/Province Level Results. Applied Psychology: An International Review. 50(2), 269-281, 2001.

[15] Straub, D., Keil, M. and Brenner, W., Testing the technology acceptance model across cultures: A three country study. Information \& Management. 33(1), 1-11, 1997.

[16] Venkatesh, V., Morris, M., Davis, G. B. and Davis, F.D., User Acceptance of Information Technology: Toward a Unified View. MIS Quarterly. 27(3), 425-478, 2003 